Jay A. Frogel and V.M. Blanco

Cerro Tololo Inter-American Observatory

\title{
1. Introduction and Summary
}

To understand the evolution of carbon and oxygen rich asymptotic giant branch (AGB) stars it is important to observationally establish the relations between parameters such as luminosity, age, and metallicity. The existence of luminous (brighter than the top of the first giant branch) AGB stars in clusters in the Magellanic Clouds (Mould and Aaronson 1979; Frogel, et al. 1980) provides a nearly ideal situation in which these relations can be determined. Although metallicities and main sequence turn-off ages are known for only a fraction of the clusters, the ranking scheme devised by Searle, et al. (1980; SWB) indicates that age and metallicity are closely related to one another.

A search has been completed and infrared photometry obtained for luminous AGB stars in 35 clusters classified by SWB. This paper discusses the bolometric luminosities of the $C$ and $M$ stars found.

\section{Observational Results}

Magellanic Cloud clusters were surveyed for $C$ and $M$ stars with a transmission grating and thin prism at the prime focus of the CTIO 4-m reflector as described by Blanco, et al. (1980, BMB). A few additional luminous red stars were identified in the cores of the most crowded clusters with the aid of multicolor images obtained with a SIT vidicon system. Infrared photometry is available, either from the literature or from new data, for all stars found. These data allow bolometric luminosities to be obtained (Frogel, et al. 1980).

Luminous carbon stars were found in 18 of the 20 clusters of SWB types IV-VI searched. With few exceptions the faintest $C$ star is more luminous than the brightest $M$ star in the same cluster. This extends considerably the result of Frogel and Cohen (1982) based on fewer clusters. We define a "transition luminosity" for a cluster as a flux average of the brightest $M$ and the faintest $C$ star. If there are no $M$ stars in a cluster, 0.7 is arbitrarily added to the bolometric 

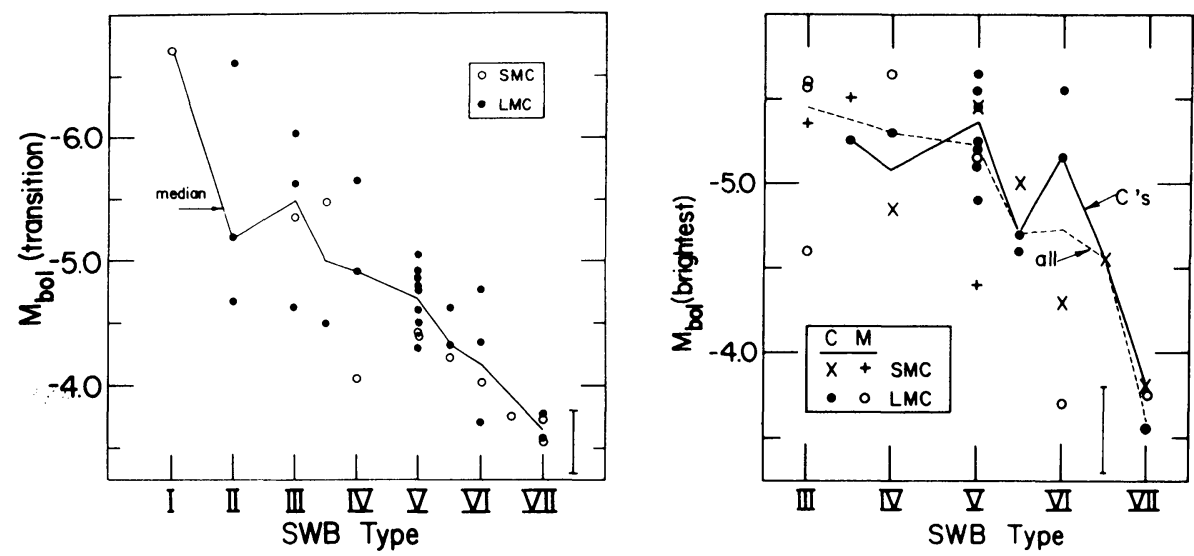

Figure 1. The C-M star transition luminosity for each of the SWB clusters surveyed. The bar at the lower right is the range in maximum luminosity observed for giants in Galactic globulars.

Figure 2. The bolometric magnitude of the brightest $C$ star (M star if a cluster has no C's) in each of the SWB clusters in our survey.

magnitude of the faintest $C$ star. If there are no $C$ stars, the transition luminosity is taken as equal to that of the brightest $M$ star. It is apparant from Figure 1 that the transition luminosity increases steadily as one goes to early (younger) SWB cluster types. A similar plot for clusters with main sequence ages (Hodge 1983) is less conclusive because such ages are available for only a small number of clusters in the sample and these tend to be systematically less than ages determined by other means for the same clusters (Hodge 1981).

The mean bolometric magnitude of the $10 \mathrm{C}$ stars in the group IV clusters is only 0.04 brighter than that of the $38 \mathrm{C}$ stars in the group $\mathrm{V}$ clusters but is 0.37 mag brighter than that of the $17 \mathrm{C}$ stars in the group VI clusters. The luminosity spread for each of the three groups of $C$ stars from these three cluster groups is 0.08-0.17 mags less than for field C stars in the Clouds (Cohen, et al. 1981). However, the combined distribution of the cluster $C$ stars is virtually identical (mean magnitude and dispersion) to that of the field stars.

Hodge (1983) noted that cluster ages derived from the luminosity of the brightest AGB star (e.g. Mould and Aaronson 1983) correlate poorly with ages derived from other techniques. Figure 2 displays the same lack of correlation. In fact, this figure suggests that for SWB groups III-VI the maximum luminosity of an AGB star is independent of age. The vertical bar in the lower right of the figure indicates the range in luminosity exhibited by the tips of the giant branches of galactic globular clusters. Tip luminosity increases with metallicity. Only long period variables in the metal rich galactic globulars exceeds 
the top of the bar (Frogel, et al. 1983). Hence the type VII clusters appear not to have luminous AGB stars, consistent with SWB's identifying them with galactic globulars.

\section{Discussion}

The dependence of the transition luminosity of SWB cluster type is qualitatively similar to Iben and Renzini's (1983, fig. 7) prediction of the dependence on age of the luminosity of the start of a star's thermally pulsing AGB phase. If ages of 0.1 and 10 Gyr are assigned to type III and VII clusters, respectively (Rabin 1982), the observed and predicted luminosities become quantitatively similar. The $S$ stars discovered by Bessell, et al. (1983) in a number of the clusters of the present sample lie close to the transition luminosities of the clusters as would be expected if they represent the first observable evidence of mixing of processed material to the surface of a thermally pulsing AGB star (as was also noted by Bessell, et al.).

From the work reported on here and from inspection of similar data presented by Bessell, et al. (1983) and Mould and Aaronson (1983) it is obvious that in a population of AGB stars which can be characterized by a single age and metallicity, carbon stars are significantly more luminous than oxygen rich stars. We have shown that a summing of cluster $C$ star luminosity functions reproduces that for Magellanic cloud field stars quite well. It is likely that a significant range in age and metallicity amongst the field star contributes to the apparent similarity of the field $C$ and $M$ star luminosity iunctions. (BMB and Cohen, et al. 1981). Furthermore, the BMB survey specifically excluded stars earlier than M5. With the inclusion of M2-4 stars (Frogel and Blanco 1983) the mean of the field M star luminosity function becomes nearly 0.5 magnitudes fainter than that for field $C$ stars. Thus, there seems to be no need to invoke nondeterministic mixing processes to explain C stars (Miller and Scalo 1982). Instead, it seems that the processes which are responsible for bringing carbon rich material to the surface of a star do so at a well defined luminosity. This luminosity is a function of stellar age and metallicity.

If the SWB cluster sequence provides an accurate age/metallicity ranking (Searle and Smith 1981), than the lack of dependence of the luminosity of the brightest AGB stars on cluster type (Figure 2) may be related to the problem of the "missing luminous C stars" (Iben 1981). The situation in Figure 2 could arise, for example, if true mass loss rates for the most luminous stars are considerably higher than normally assumed. In any case, Figure 2 is not consistent with the use of these luminous stars as age indicators.

Finally, on the basis of these data we consider it unlikely that luminous $C$ stars can be converted back into luminous $M$ stars (Iben 1981). If such a fate befell a significant fraction of the most luminous $C$ stars, then the almost complete lack of overlap in luminos- 
ity for the M's and C's in a cluster would require that the bornagain M star be of much lower luminosity than its predecessor. This would violate the core-mass luminosity relationship.

\section{REFERENCES}

Bessell, M.S., Wood, P.R., and Lloyd Evans, T.: 1983, M.N.R.A.S. 202, p. 59 .

Blanco, V.M., and Frogel, J.A.: 1983, Ap. J., to be submitted.

Blanco, V.M., McCarthy, M.F., and Blanco, B.: 1980. Ap. J. 242, p. 938.

Cohen, J.G., Frogel, J.A., Persson, S.E., and Elias, J.H.: 1981, Ap. J. 249, p. 481 .

Frogel, J.A. and Cohen, J.G.: 1982, Ap. J. 253, p. 580.

Frogel, J.A., Cohen, J.G., and Persson, S.E.: 1983, Ap. J., in press. Frogel, J.A., Persson, S.E., and Cohen, J.G.: 1980, Ap. J. 239, p. 495. Hodge, P.: 1981, in IAU Coll. 68, Philip, A.G.D. and Hayes D.S., eds. L. Davis Press, p. 205.

Hodge, P.: 1983, Ap. J. 264, p. 470.

Iben, I., Jr.: 1981, Ap. J. 246, p. 278.

Iben, I., Jr., and Renzini, A.: 1983, Ann. Rev. Astr. Ap., in press.

Miller, G.E., and Scalo, J.M.: 1982, Ap. J. 263, p. 259.

Mould, J., and Aaronson, M.: 1979, Ap. J. 232, p. 421.

Mould, J., and Aaronson, M.: 1983, Ap. J., in press.

Rabin, D.: 1982, Ap. J. 261, p. 85.

Searle, L., and Smith, H.A.: 1981, in IAU Cool. 68, Philip, A.G.D. and Hayes, D.S., eds., L. Davis Press, p. 201.

Searle, L., Wilkinson, A., and Bagnuolo, W.: 1980, Ap. J. 239, p. 803.

\section{DISCUSSION}

Mould: One of the sources of scatter in Figure 2 mist be the stochastic population of the AGB. Less massive clusters have a lower probability of approximating a star truly marking the AGB tip. Secondly, one should note that the luminosity function of Long Period Variables in the Magellanic Clouds and the luminosity function of photometrically selected red giants show that some stars with bolometric magnitude -6 are present. Their numbers are reduced but not to zero.

Froge1: I agree that the Long Period Variables being found in the Clouds are quite luminous. The problem is their scarcity. 